\title{
Making adaptive cruise control (ACC) limits visible
}

\author{
Bobbie D. Seppelt*, John D. Lee \\ Department of Mechanical and Industrial Engineering, The University of Iowa, 3131 Seamans Center, IA 52242, USA \\ Received 16 January 2006; received in revised form 25 September 2006; accepted 5 October 2006 \\ Communicated by C. Burns \\ Available online 20 November 2006
}

\begin{abstract}
Previous studies have shown adaptive cruise control (ACC) can compromise driving safety when drivers do not understand how the ACC functions, suggesting that drivers need to be informed about the capabilities of this technology. This study applies ecological interface design (EID) to create a visual representation of ACC behavior, which is intended to promote appropriate reliance and support effective transitions between manual and ACC control. The EID display reveals the behavior of ACC in terms of time headway (THW), time to collision (TTC), and range rate. This graphical representation uses emergent features that signal the state of the ACC. Two failure modes - exceedance of braking algorithm limits and sensor failures - were introduced in the driving contexts of traffic and rain, respectively. A medium-fidelity driving simulator was used to evaluate the effect of automation (manual, ACC control), and display (EID, no display) on ACC reliance, brake response, and driver intervention strategies. Drivers in traffic conditions relied more appropriately on ACC when the EID display was present than when it was not, proactively disengaging the ACC. The EID display promoted faster and more consistent braking responses when braking algorithm limits were exceeded, resulting in safe following distances and no collisions. In manual control, the EID display aided THW maintenance in both rain and traffic conditions, reducing the demands of driving and promoting more consistent and less variable car-following performance. These results suggest that providing drivers with continuous information about the state of the automation is a promising alternative to the more common approach of providing imminent crash warnings when it fails. Informing drivers may be more effective than warning drivers.
\end{abstract}

(C) 2006 Elsevier Ltd. All rights reserved.

Keywords: EID; ACC; Reliance; Automation failure; Collision warnings

\section{Introduction}

Automation is fundamentally changing the role of people in many systems, and driving is no exception. An increasing number of vehicles are being equipped with adaptive cruise control (ACC). This technology is substantially more complex than conventional cruise control; ACC adjusts the brake and/or throttle, within limited ranges, to maintain a constant headway from any vehicle that intrudes upon the path of the driver's vehicle. While ACC provides a potential safety benefit in helping drivers maintain a constant speed and headway (Davis, 2004), as with other types of automation, there is the potential for misuse and disuse (Parasuraman and Riley, 1997).

\footnotetext{
*Corresponding author. Tel.: + 13193840514 ; fax: + 13193356801 .

E-mail addresses: bseppelt@engineering.uiowa.edu (B.D. Seppelt), jdlee@engineering.uiowa.edu (J.D. Lee).
}

For ACC to be effective, drivers need to understand the capabilities of the technology, which include braking and sensor limitations. Based on this understanding, they must be able to intervene when a given situation exceeds ACC capabilities. However, drivers have difficulties in understanding how ACC functions (Stanton and Marsden, 1996). As a result, they tend to rely on the system inappropriately. For instance, Nilsson (1995) showed that drivers failed to intervene when approaching a stopped queue of vehicles because they believed that the ACC could effectively respond to the situation. Stanton et al. (1997) introduced an unexpected acceleration into the ACC system during routine driving conditions, which resulted in a collision $33 \%$ of the time. Whether or not drivers can respond effectively when automation fails depends on their understanding of the type of failure that occurs and the context in which it occurs (Lee and See, 2004). In Seppelt et al. (2005a), two failure types were introduced within 
specific driving contexts to determine their effect on ACC reliance. In conditions of rain, the signal continuity of the ACC sensors was degraded, and in conditions of heavy traffic, the braking limits of the ACC system were exceeded. Results showed that the failure type influenced drivers' reliance on ACC, with drivers relying more on ACC in traffic periods than in rain periods. Reliance on ACC in traffic periods led drivers to disengage from the driving task, evident from an increased response time to lead vehicle (LV) braking. This study revealed the need to display information regarding sensor performance and operational limits of ACC to support drivers in their understanding of such systems.

To ensure safe and effective use, ACC limits of operation should be identifiable and interpretable (Goodrich and Boer, 2003). One approach to help drivers detect and respond to these limits is to match the limits of the ACC algorithm to the natural boundaries drivers use to switch between car-following and active braking behaviors, as defined by environmental cues [e.g. time headway (THW) and time to collision (TTC)]. Essentially, this means matching the function of the ACC to the way drivers perform the task of following other vehicles (Goodrich and Boer, 2003). Individual differences in driving behavior, however, would require some degree of tuning of the ACC algorithm to individual drivers.

Representing ACC limits is another approach. A functional representation could help drivers to develop accurate and reliable expectations of the automation's abilities. Another reason such an approach may benefit drivers is that ACC failures provide few salient cues - the THW and TTC cues often change gradually, and drivers' responses may be delayed because the initial changes might not exceed their perceptual thresholds (Hills, 1980; Hoffmann and Mortimer, 1994, 1996). Providing drivers with a better representation of ACC behavior may enhance understanding of ACC, encourage more effective monitoring, and promote more appropriate reliance.

This study applied ecological interface design (EID) to create a visual representation to support appropriate reliance on ACC. EID provides a theoretical structure to guide interface design of complex systems, such as process control plants. It identifies environmental constraints that define the information requirements for both normal operations and unexpected situations (Vicente and Rasmussen, 1992; Vicente, 1999). EID helps identify critical variables for control, and guides display of such information in a way that is consistent with people's perceptual and cognitive capabilities. Although, it has been successfully applied to several diverse domains (Vicente, 2002), EID has not been systematically applied and tested in the driving domain. However, EID promises to identify and represent information needed to support drivers (Stoner et al., 2003).

\subsection{EID for adaptive cruise control}

EID was used to develop a display that maps limits of the ACC to a graphical representation that provides drivers with continuous information regarding the state of the ACC. This is particularly important when the ACC is confronting situations for which it was not designed and when sensors fail. In both situations, drivers might tend to rely on the system inappropriately. Dzindolet et al. (2003) showed that providing participants with information on why an automated aid could fail led to more appropriate reliance than that demonstrated by participants not provided with this information. In addition, when operators were provided with continuous feedback on the aid's performance, their reliance on the automation was more appropriate. These provisions are consistent with using EID to convey the limits and dynamic state of the ACC. To the authors' knowledge, no research has addressed how to convey the limits and performance of ACC to drivers to enhance appropriate reliance.

Another design philosophy that might guide the information provided to drivers regarding the limits of the ACC system, the display-by-exception approach, displays information only when the automation fails and requires immediate attention (Palmer and Abbott, 1994). Such an approach argues that continuous information, as provided in the EID display, will distract drivers from attending to the driving task, potentially leading to unsafe driving situations. However, unlike discrete warnings associated with the display-by-exception approach, a continuous display allows drivers to observe the automation's behavior in a variety of situations. EID may enable drivers to develop accurate expectations of the automation's abilities and to associate various display configurations with the evolution of non-hazardous and hazardous situations. The expectation is that the continuous display will provide drivers with a more complete mental representation of the ACC's behavior, allowing them to identify and respond to abnormal behavior prior to and more effectively than with a warning system.

The EID display for this study provides continuous information on the relationship between the driver's vehicle and the LV using an object whose shape and position continuously changes to reflect the speed and distance between the two cars. Relevant variables of TTC, THW, and range rate (i.e. relative velocity between vehicles) animate the display. These variables were derived from a cognitive work analysis (CWA) of the control tasks associated with headway maintenance, situation assessment, and collision avoidance (Seppelt et al., 2005b), which identified the variables as constraints within the driving environment. THW, TTC, and range rate indicate the distance $(\mathrm{m})$ and speed $(\mathrm{m} / \mathrm{s})$ that defines the state of the two vehicles. These variables provide the basic information needed to control speed and distance in highway driving (Fancher et al., 2001). Each variable is mapped onto the display in the following manner (see Fig. 1):

$T H W=$ distance $_{\text {velocity }}$ driver vehicle: Defines the vertical position of the shape within the display. As THW decreases, the shape moves closer to the bottom of the display, and as THW increases, the shape moves to the top 

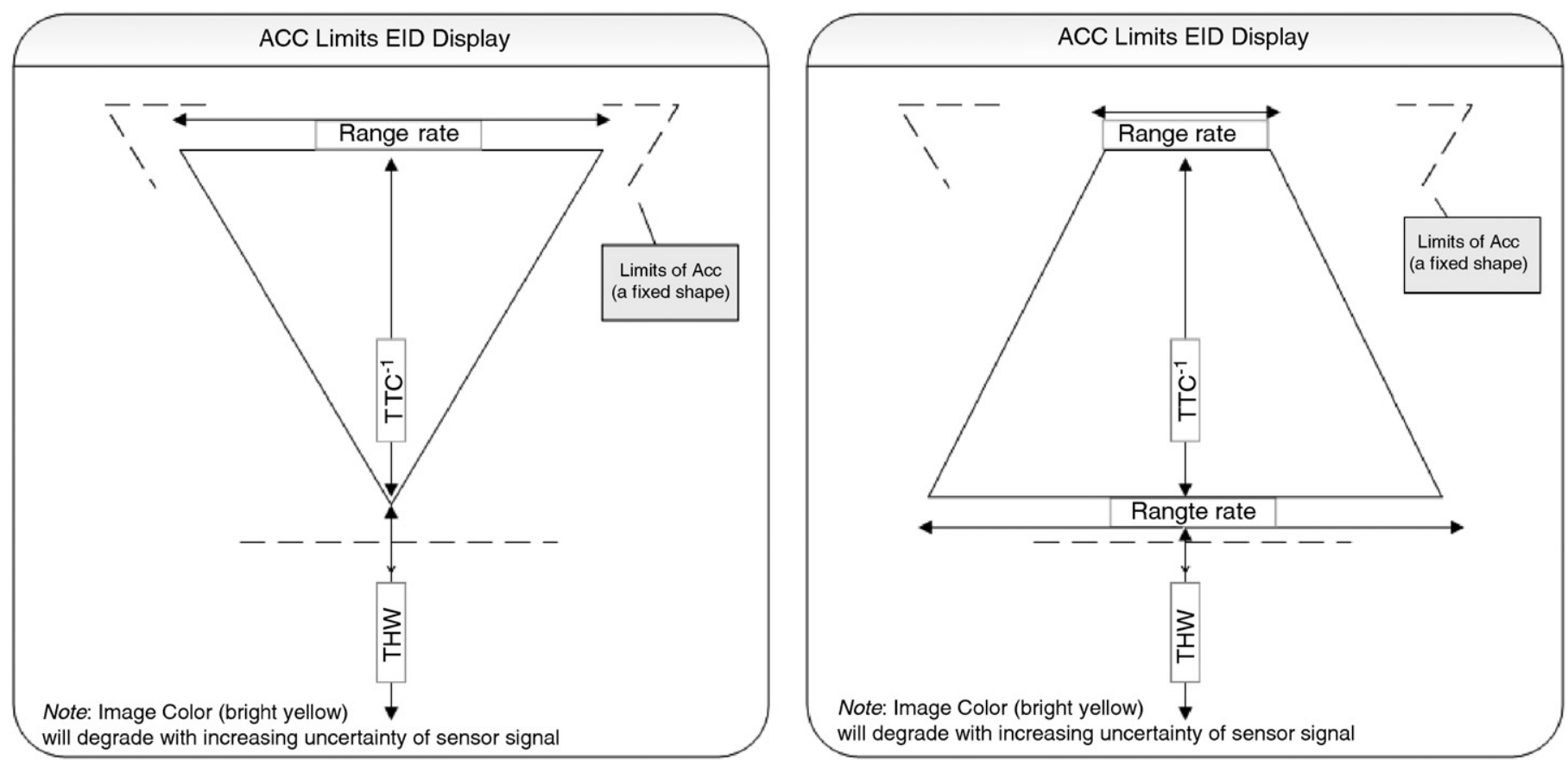

Fig. 1. EID display: (a) triangle or yield shape and (b) trapezoid shape.

of the display. At a THW $>7.0 \mathrm{~s}$ the shape moves off the display, alerting drivers that THW is no longer detectable given the large time distance between their vehicle and the LV.

$T T C^{-1}=\left(\text { distance }\left(\text { velocity }_{\text {driver }} \quad \text { vehicle }- \text { velocity }_{\mathrm{LV}}\right)\right)^{-1}$ : Defines the height of the object within the display. As TTC decreases (or as the rate of closure increases), the shape expands vertically (at $\mathrm{TTC}=0$, the point of collision, the shape is maximally expanded to the height of the display), and as TTC increases, the shape contracts vertically. At a TTC $>20 \mathrm{~s}$, the shape contracts to a minimum fixed height.

Range rate $=$ velocity $_{\text {driver vehicle }}-$ velocity $_{\mathrm{LV}}$ : Defines the width of the object within the display. As range rate increases, the shape expands horizontally, and as range rate decreases, the shape contracts horizontally. This effect, coupled with the vertical movement associated with the TTC $^{-1}$ variable, creates a looming effect and indicates potentially hazardous braking events.

Two distinct shapes emerge from the combination of variables: a triangle and a trapezoid; these shapes provide two general categories of situations, those (potentially) hazardous and those non-hazardous, respectively, for drivers to better understand the behavior of the ACC over time for a variety of situations:

Triangle or yield shape (see Fig. 1a): The triangle shape emerges when the driver's vehicle is approaching the LV or when the two vehicles are traveling at the same speed (i.e. range rate is greater than or equal to zero). This shape is intended to keep drivers in a state of readiness; the yield shape indicates the need to drive with caution.

Trapezoid shape (see Fig. 1b): The trapezoid shape emerges when the LV is driving faster than the driver (i.e. range rate is less than zero). This shape is intended to appear non-threatening and distinctly different than the shape that appears when the driver is approaching the LV.

The transition between the yield shape and the trapezoid shape occurs seamlessly through the range rate variable. As the driver's vehicle approaches a speed equivalent to that of the $\mathrm{LV}$, the range rate axis in the yield shape contracts until a set distance is reached, which represents equal speeds (see the top trapezoid side in Fig. 1b). This set distance defines the top axis of the trapezoid shape. Note that the top axis does not contract to a point to keep the shape from reducing to a straight line. At the instant the LV begins to pull away, the top axis remains fixed while the bottom axis expands horizontally from its single axis point, thus creating the trapezoid shape seen in Fig. 1b. Fig. 2 shows an example of how the shape transitions as equivalent speeds are reached between the driver's vehicle and the LV, and as the LV then pulls away from the driver's vehicle. The reverse of this movement takes place when the driver's speed increases towards a speed equivalent to that of the LV.

Dashed lines in the display convey the limits of the ACC's braking authority and provide visual "safety boundary" references for when the ACC is not engaged. The dashed line near the bottom of the display indicates a minimum $(\sim 1.5 \mathrm{~s})$ THW boundary of the ACC's braking limits and of a safe following distance in manual control. When the shape exceeds the two triangular reference lines (seen in Fig. 1a and b), the ACC's braking power has reached the $0.28 \mathrm{~g}$ limit; in manual control this displayed rate of closure to the LV informs of an imminent collision situation. These reference lines remain fixed, representing the fixed braking limits of the ACC, and the shape moves 

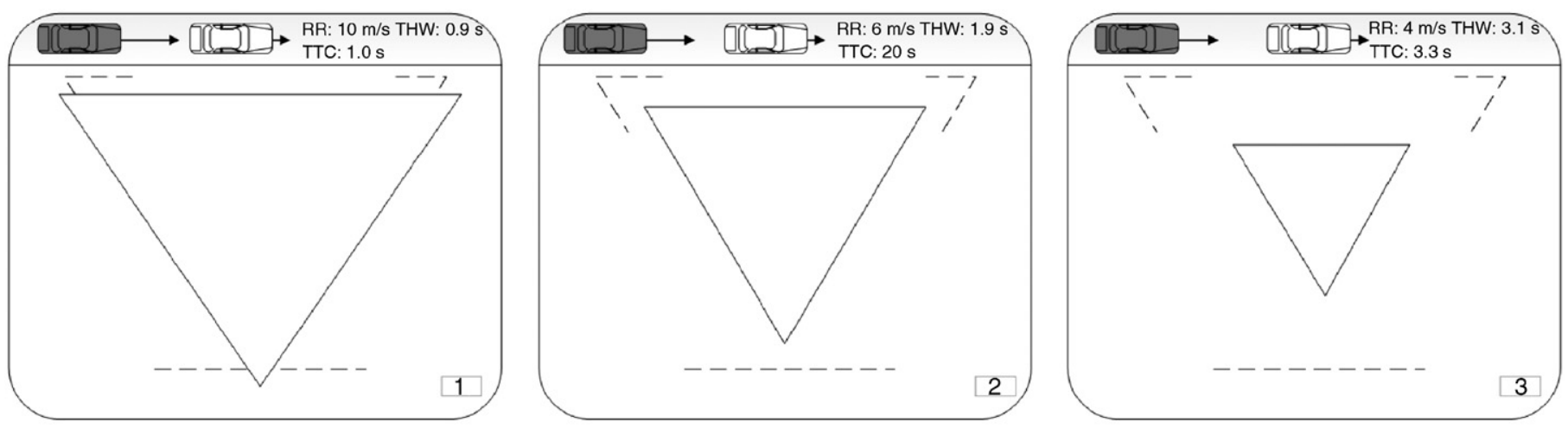

Time
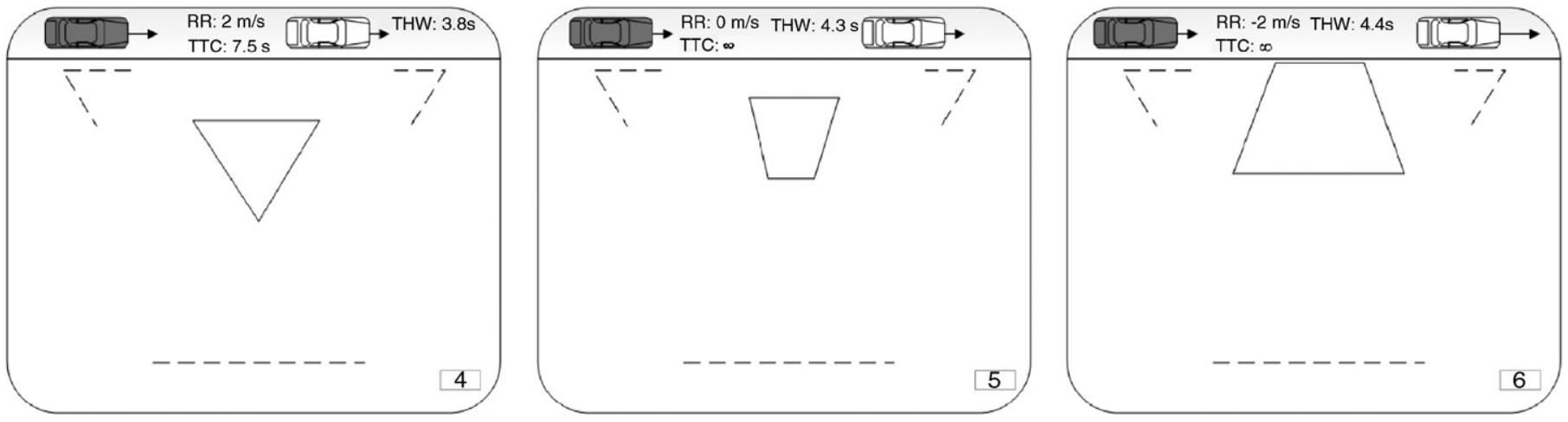

Fig. 2. Example behavior of EID display over time when a driver's vehicle approaches a LV (slides 1-3), equivalent speeds are reached (slide 4), and the LV then pulls away from the driver's vehicle (slides 5,6). Kinematics description between the driver vehicle and LV, and range rate (RR), THW, and TTC variables are noted in the slide titles.

in relation to the fixed lines. Differences in time to reach the braking limits with varied speed differentials and THWs are reflected in the rate of change of the shape. For example, if THW is shorter than $1.5 \mathrm{~s}$ and the LV brakes, the TTC and range rate variables will change more rapidly - and thus the braking limits will be exceeded more rapidly - than if the THW were equal to or longer than $1.5 \mathrm{~s}$. The dashed triangular lines represent the situation where $\mathrm{TTC}^{-1}$ and range rate are hazardous - when the shape exceeds their position vertically and horizontally, respectively. All hazardous situations are thus bounded by these lines (i.e. the lines do not form a closed shape) in that either a horizontal or vertical movement of the shape beyond the reference lines indicates that the $0.28 \mathrm{~g}$ braking limit of the ACC has been reached.

The display conveys sensor degradation in two ways: (1) color dilution and (2) update rate failure. As the intensity of the rain increases, the color of the shape diminishes from a bright yellow, the normal operating color, to a light yellow to a light gray during the highest rain levels. The color dilution, in degrading the image, provides drivers with a continuous measure of the reliability of the displayed information (Finger and Bisantz, 2002). When a sensor failure occurs, a result of the rain temporarily attenuating the radar signal, the displayed information is not updated, creating a momentary freezing of the shape for the duration of the sensor failure. The shape then "jumps" to the appropriate position following the sensor failure.

Exceedance of braking algorithm limits and sensor degradation represent two qualitatively different types of ACC failures. The braking algorithm exceedances are event-based failures that occur in response to LV braking. The sensor degradation failures are condition-based failures that result from the interaction of the environment with the operation of the ACC. Both failures are evident from the decrease in THW between the driver's vehicle and the LV when the failure occurs. The factor affecting the capability of the automation when the braking algorithm exceedance occurs, the LV braking event, is visible in the environment, providing a certainty of failure. However when sensor degradation occurs, the behavior of the LV is unconnected to the failure, making it a non-confirmatory cue of automation performance. The rain, based on its intensity and duration, is a probabilistic cue of failure.

\subsection{Purpose}

The purpose of this experiment was to assess whether an EID display can promote appropriate reliance and support 
effective transition between manual and ACC control in the context of two failure types: sensor degradation and exceedance of braking algorithm limits. This paper also examined the benefits of the EID display to manual control; it was expected that the EID display would make drivers more sensitive to changes in the driving situation and so support more consistent car-following behavior when drivers did not have the ACC engaged. A secondary task was included to measure resources not committed to vehicle control (Wickens, 1992). It was expected that the EID display would diminish the demands associated with monitoring ACC and reduce the processing demands associated with assessing the ACC state, thereby freeing resources to respond to the secondary task.

\section{Method}

This study addressed the effect of providing continuous information on automation capability in two different driving situations, which produced qualitatively different failure types. Participants interacted with an ACC system, and were responsible for maintaining longitudinal control (either manually or with the ACC control) and for using the ACC system based on its appropriateness in the particular driving context.

\subsection{Participants}

Twenty-four participants (17 male, 7 female) between the ages of 25 and $40(M=30.8, \mathrm{SD}=4.8)$ were recruited from the Iowa City community. All were native English speakers with active driver's licenses. Participants were recruited as volunteers and paid $\$ 15 / \mathrm{h}$ for approximately $2.5 \mathrm{~h}$ of participation. To encourage drivers to engage the ACC when available, participants who maintained a THW of $1.5 \pm 0.15 \mathrm{~s}$ for the drive received a $\$ 5$ bonus. For less precise THW maintenance, drivers received $\$ 4$ when they deviated from a $1.5 \mathrm{~s}$ THW by $0.16 \mathrm{~s}$ to $0.30 \mathrm{~s}, \$ 3$ when they deviated by $0.31-0.50 \mathrm{~s}, \$ 2$ when they deviated by $0.51-0.70 \mathrm{~s}, \$ 1$ when they deviated by $0.71-1.0 \mathrm{~s}$, and $\$ 0$ when they deviated beyond $1.0 \mathrm{~s}$. An additional bonus (up to \$5) was awarded if participants performed well on the secondary task.

\subsection{Apparatus}

Data were collected at $60 \mathrm{~Hz}$ using a fixed-base driving simulator with a $50^{\circ}$ field of view, full instrumentation with functional gauges, a force-feedback steering wheel, and a surround-sound audio system. The fully textured graphics were delivered at a $60-\mathrm{Hz}$ frame rate at $1024 \times 768$ resolution. Rain was simulated on the forward screen, visible as falling raindrops superimposed on the driving environment. This rain was audible through the surroundsound speakers. Windshield wipers were not used. A 7-in head-down display with a $640 \times 480$ pixel screen displayed the EID display. This display was mounted on the dash of the car, approximately $8^{\circ}$ horizontally and $11^{\circ}$ vertically from the driver's line of sight.

\subsection{Driving environment overview}

Separate drives containing either rain or traffic periods were created. The two types of drives were independently configured to create conditions appropriate for the two different ACC failure types. In drives with rain periods, the driving environment consisted of two-lane rural roadways. For drives with traffic periods, the environment consisted of four-lane interstate freeways. Participants drove the roads in both directions, encountering oncoming traffic at a rate of three to five cars per minute; this moderate level of traffic was also present in the passing lane for the four-lane roadway.

\subsection{Driving task}

Each participant drove four, 20-min drives, two with manual control and two in which ACC was available (i.e. participants chose when and if to engage the ACC). When ACC was not in use, drivers were instructed to maintain a 1.5 -s headway to the LV, which varied in speed throughout the drive. Of the two drives with manual control and with ACC, respectively, one included rain periods and the other traffic periods. The two rain drives each had two 3-min and one 6-min periods of rain. Two-min periods with no rain separated the rain periods, providing a baseline period for the LV-following task in which the ACC operated normally. Similarly, the two drives with periods of intense traffic consisted of two 3-min and one 6-min periods, separated by 2-min baseline periods.

During the rain periods, the LV velocity followed a seemingly random pattern defined by the sum of two sine waves (amplitude, frequency, and phase for the first and second sine waves were $5,0.25$ and 5 , and $3,0.05$ and 0 , respectively). The duration of each $8-12 \mathrm{mph}(13-19 \mathrm{~km} / \mathrm{h})$ increase and subsequent decrease in LV velocity was between 10 and $15 \mathrm{~s}$. This gradual increase and decrease in $\mathrm{LV}$ velocity did not require the driver to respond when the ACC was engaged, as the automation maintained a set $1.5 \mathrm{~s}$ THW. In manual control, however, drivers were required to modulate the accelerator or brake to maintain this $1.5 \mathrm{~s}$ THW. In the traffic periods, the LV velocity varied according to a pattern of rapid deceleration and acceleration, with a change in velocity of between 10 and $16 \mathrm{mph}(16$ and $26 \mathrm{~km} / \mathrm{h})$. The deceleration and acceleration associated with these changes in velocity were separated by $1-5 \mathrm{~s}$. A velocity change occurred every $9-15 \mathrm{~s}$. In the rain periods, the variations in LV behavior were introduced only to keep drivers engaged in maintaining the $1.5 \mathrm{~s}$ THW. In the traffic periods, although the variations in LV speed were intended to keep drivers engaged in the LV following task, they were also designed to exceed the braking capacity of the ACC, resulting in events in which the ACC failed to maintain the set 
headway. These failure events are discussed in the following section.

\subsection{Adaptive cruise control}

The ACC system operated when the vehicle was traveling between $20 \mathrm{mph}(32 \mathrm{~km} / \mathrm{h})$ and $85 \mathrm{mph}(137 \mathrm{~km} /$ h). If no vehicle was detected in front of the participant vehicle, the ACC operated like a conventional cruise control system, maintaining a set speed of $55 \mathrm{mph}$ $(89 \mathrm{~km} / \mathrm{h})$. If a slower moving vehicle was in front of the participant vehicle, the ACC reduced vehicle speed to maintain a $1.5 \mathrm{~s}$ THW, using a maximum of $33 \%$ of the vehicle's braking power, or $0.28 \mathrm{~g}$. Drivers pressed an "On" button located on the steering wheel to engage the ACC. To disengage the system, drivers could either press an "Off" button located on the steering wheel or depress the brake pedal.

In the traffic periods, the magnitude of the LV velocity changes increased over time, peaked at $90 \mathrm{~s}$ (or $180 \mathrm{~s}$ during the 6-min periods), and then decreased in a symmetrical fashion. Traffic density, which increased from approximately 6 to 19 vehicles $/ \mathrm{km}$ at the peak 90 -s mark, influenced the LV velocity such that an increase in density corresponded with an increased instability of traffic flow, creating situations that required larger variations in LV velocity. As the braking of the LV became more severe, the $0.28 \mathrm{~g}$ braking limit of ACC was exceeded, compromising the ability of the ACC to maintain a $1.5 \mathrm{~s}$ headway.

In the rain periods, the degree of fog and rain also increased over time, peaked at $90 \mathrm{~s}(180 \mathrm{~s}$ during the 6-min periods), and then decreased symmetrically; the sight distance similarly decreased from 1000 to $10 \mathrm{~m}$ at $90 \mathrm{~s}$ ( $180 \mathrm{~s}$ for the 6-min periods) then gradually increased back to $1000 \mathrm{~m}$. Rain degraded the ability of the radar to detect vehicles ahead, as it attenuated the sensor sensitivity, masking the sensors and causing them to fail temporarily. With no LV detected, vehicle speed increased to $55 \mathrm{mph}$. As the intensity of the rain increased, the sensors failed for longer periods and failed more frequently. The momentary failure of the sensors to detect vehicles ahead compromised the ability of the ACC to maintain a $1.5 \mathrm{~s}$ headway. (Note: The parameters and limitations of the ACC system were modeled after a 2004 Mercedes-Benz E-Class Distronic ACC system.)

Sensor failure durations of $0.5,1.8,3.35$, and $5.27 \mathrm{~s}$, and braking algorithm exceedances of 1.0, 2.0, 3.0, and $5.0 \mathrm{~s}$, coded as level 1, level 2, level 3, and level 4 of ACC failure, corresponded to approximate THWs of 1.5, 1.3, 1.1, and $0.9 \mathrm{~s}$ when a driver did not intervene and disengage the ACC. These THW consequences are all within a safety margin to react to unexpected events (Hoedemaeker, 2000; Fancher et al., 2001). TTC, sampled at the end of each failure, indicates the collision likelihood if the vehicles maintained the same velocities. In the rain periods, TTC for failure levels $1,2,3$, and 4 were approximately 20,14 ,
10 and $4.5 \mathrm{~s}$, respectively, and in the traffic periods, 8.5, 9, 5.5 and $3.5 \mathrm{~s}$, respectively.

For ACC rain and traffic drives, the two 3-min and one 6min periods included a total of 44 failures per drive, which consisted of 16 level-1, 16 level-2, 8 level-3, and 4 level-4 failures. Each sensor failure and braking algorithm exceedance occurred only if the ACC was engaged. It is important to note that in the traffic drives, the variations in $\mathrm{LV}$ velocity exceeded the braking capacity of the ACC. The braking algorithm failed to accommodate the rapid decreases in velocity; these decelerations were $0.90 \mathrm{~g}$ for $0.5 \mathrm{~s}, 0.90 \mathrm{~g}$ for $0.5 \mathrm{~s}, 0.91 \mathrm{~g}$ for $0.7 \mathrm{~s}$ and $0.75 \mathrm{~g}$ for $1.0 \mathrm{~s}$ for failure levels 1,2 , 3 and 4, respectively. However, in the rain drives, the variations in LV velocity did not exceed the braking capacity of the ACC. Failures resulted from sensor degradation, which occurred independently of the LV's behavior.

In each 3-min rain and traffic period, 11 failures occurred - five randomly distributed in the initial $90 \mathrm{~s}$ of the period, one during the peak $90 \mathrm{~s}$ of the period, and the remaining five arrayed symmetrically in the final $90 \mathrm{~s}$. The THW consequences of the failure events increased, peaked at $90 \mathrm{~s}$, and then decreased symmetrically over the 3-min with the following failure level sequence: 1, 1, 2, 2, 3, 4, 3, 2, $2,1,1$. The level 1 failures are barely perceptible and the level 4 failures represent unambiguously threatening situations. For the 6-min periods, the number of failures doubled, for a total of 22 , with the same pattern of increasing and decreasing failure levels, except that each failure occurred twice. This pattern of increasing and decreasing failure levels is intended to model the real-world degradation of the sensors or increasing severity of braking responses that would occur with an increase in rain and traffic, respectively.

\subsection{Experimental design}

A four-factor $\left(2^{3} \times 4\right)$ experimental design, mixed between and within subjects, considered two levels of failure type, sensor degradation and exceedance of braking algorithm limits (within the rain and traffic periods, respectively); two levels of automation, manual and ACC control; two levels of display type, EID display and a baseline condition (no display); and four levels of failure level. Display type was introduced as a between-subjects variable. The EID display is not compared to the icons that current production vehicles use to display ACC state, as many production vehicles only display static information that would not have varied during the experiment, such as set speed and set gap or THW values. This static information is not relevant for the participants' tasks and so is not substantially different from the included nodisplay baseline. The order of conditions was counterbalanced across drivers.

\subsection{Procedure}

Upon arriving, participants completed an informed consent form and then drove a short, 5-min drive; this 
drive acclimated participants to the driving simulator controls and environment. Participants drove an additional 5 -min practice drive, which introduced them to the ACC, allowing them to become familiar with the ACC system, the controls used to engage and disengage the system, and the $1.5 \mathrm{~s}$ THW it maintained. In both the practice and experimental drives, participants were instructed to maintain a $1.5 \mathrm{~s}$ THW to the LV for the duration of the drive. Participants were reminded that the ACC maintained a precise $1.5 \mathrm{~s}$ THW to the LV. Participants were also instructed to drive safely, to stay aware of the vehicles ahead, and to avoid unnecessary braking.

Each participant was randomly assigned to a display condition. For the drives in which ACC was available, participants were instructed to engage and disengage ACC in accordance with their assessment of the appropriateness of using the system. They were advised to use the ACC only if the roadway conditions allowed safe driving at a steady speed. They were also informed of the ACC system's maximum braking power of $33 \%$ or $0.28 \mathrm{~g}$. Further, instructions on the EID display informed participants that the shape conveyed speed and distance information. The two shapes and reference lines were noted, but the variables that animated the display and the meaning of the reference lines were not described. The EID display operated continuously for both the manual and ACC drives.

Throughout each drive, a continuous secondary task required listening and verbally responding to messages related to upcoming restaurants (Reyes and Lee, 2004). As this task was auditory and verbal, it did not directly interfere with visual sampling of the EID display. Each message presented information about the cost, quality, and wait time for each of three different restaurants. Six messages were presented during each drive, and each message was followed by six questions based on the information presented. After each message, drivers verbally indicated when they were ready for the questions to begin. Answers were spoken out loud and took the form of a restaurant name. Drivers were required to answer all questions. In the description of the secondary task, drivers were reminded to drive safely and to listen to the content of the messages and the corresponding questions throughout the entire drive. Participants were debriefed and compensated following the experimental drives.

\subsection{Dependent variables}

Automation reliance is often discussed in terms of a binary process of use. However, reliance is a complex process affected by multiple factors, some of which include automation reliability, trust in the automation, selfconfidence in one's own abilities, task complexity, risk, and fatigue (Riley, 1994). Reliance may therefore be more aptly described as a graded process whereby these underlying factors influence the degree of reliance. In this paper, both definitions of reliance are considered. Automation reliance is first measured as percent of time the ACC was engaged. Percent reliance was calculated from the start to the end of each automation failure for each participant (i.e. the percentage of time a participant had ACC engaged during each failure). Percent reliance was also calculated during all non-failure periods.

The second measure of automation reliance is driver intervention. Two measures describe the effect of failure type on driver intervention. Brake reaction time (RT), defined as the time from the start of a failure to when a driver depresses the brake more than $5 \%$ of the total brake pedal range, specifies the amount of time it takes for a driver to respond to an ACC failure. The second descriptor of the effect of failures on driver response is THW at brake response. It is important to note that brake RT includes only those responses in which drivers depressed the brake pedal, while percent reliance includes both responses in which drivers depressed the brake pedal and those in which they pressed the "Off" button. Percent reliance also includes those instances where drivers engaged and disengaged ACC multiple times during a failure. Measures of brake RT and percent reliance provide complementary perspectives of automation reliance - the correlation of mean percent reliance and brake $\mathrm{RT}$ in rain and traffic periods were not statistically significant, $r(13)=.47$, $p=.08, r(19)=.33, p=.14$, respectively.

Two measures describe the potential benefit of the EID display during manual control: brake RT to a LV deceleration, and THW at brake response. Brake RT, defined as the time from the start of a decrease in the LV's velocity to when a driver depresses the brake more than $5 \%$ of the total brake pedal range, specifies the amount of time it takes for a driver to respond to a LV deceleration. Note that this measure differs from the brake RT for driver intervention only in terms of the event to which the driver responds (i.e. an automation failure versus a LV deceleration). For the secondary task, dependent variables include question RT and response accuracy.

\section{Results and discussion}

Results focus on whether, in the context of ACC failures, drivers rely on the ACC appropriately and thus intervene effectively. Of particular interest is the degree to which the EID display modulated automation reliance. A secondary consideration is the benefit of the EID display during manual control. The 2-min baseline periods were included only in the automation reliance analysis. Rain and traffic drives were analysed separately as they are qualitatively different. The ACC drives were divided into engaged (ACC on) and not-engaged (ACC off) portions. The data were analysed using SAS 9.0 software, specifically, the mixed linear model (MIXED) procedure with compound symmetry covariance structure. $F$ statistics are reported from repeated measures ANOVAs that included display type as a between-subject variable and automation level and failure level as within-subject variables. An $\alpha$-value of .05 was used as the criterion for statistical significance. The 
figures show standard error (SE) bars of \pm 1 SE for each mean.

\subsection{Automation reliance}

Fig. 3 shows mean percent reliance on ACC across subjects, over the rain (Fig. 3a) and traffic (Fig. 3b) periods by display type and failure level. Short and long periods (i.e. 3 and 6 -min rain/traffic periods, respectively) are combined across each failure level. Note that two points are plotted per display for each failure level - the top point reflects the mean percent reliance for the first half of the period, and the bottom point reflects the mean percent reliance for the second half of the period. In both the rain and traffic periods, an increase in failure level resulted in a decrease in reliance, $F(3,66)=4.02, p=.011, F(3,66)=$ $8.24, p<.0001$, respectively. For any given level of reliability, drivers' reliance in both the rain and traffic periods was greater when the automation was degrading compared to when it was recovering, $F(1,22)=38.46$, $p<.0001, F(1,22)=66.86, p<.0001$, respectively. Essentially, drivers relied on ACC less during the second half of
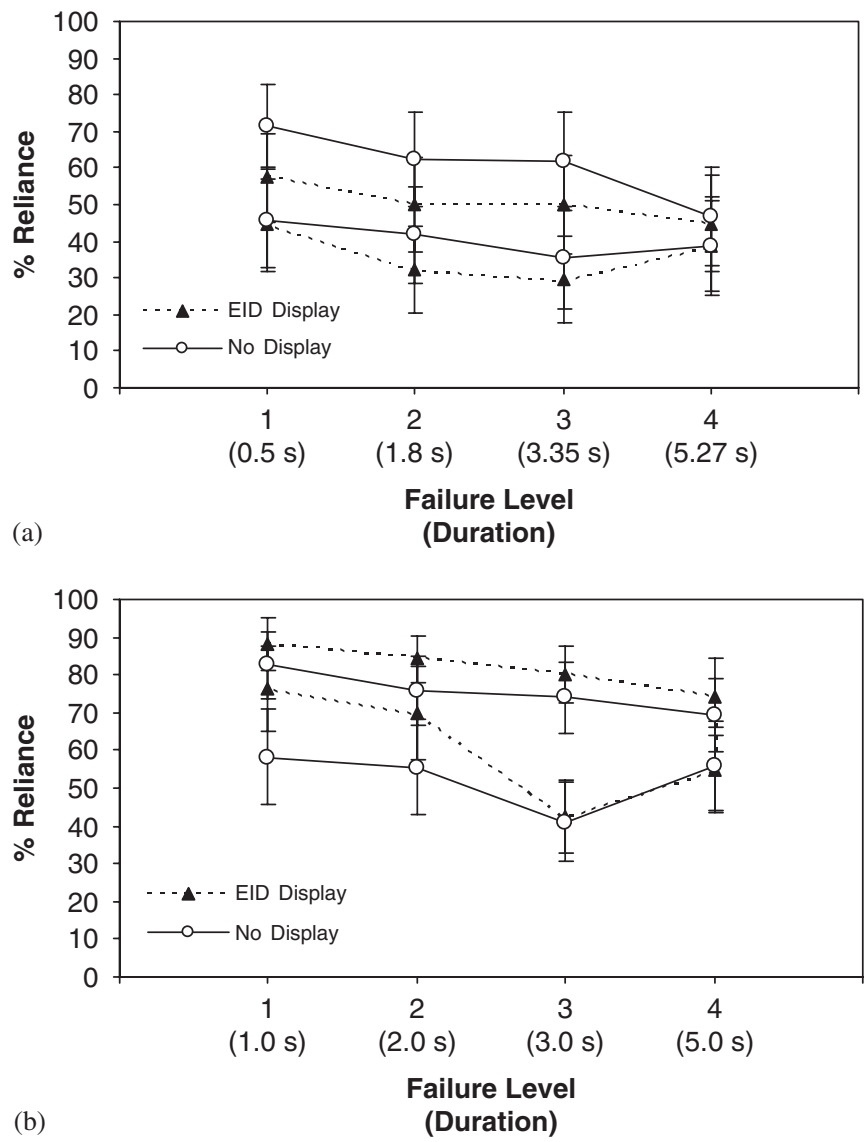

Fig. 3. Percent reliance versus ACC failure level for EID display and no display conditions in (a) rain periods, and (b) traffic periods. For each failure level, by display, the top point is the mean percent reliance for the first half of the period and the bottom point is the mean percent reliance for the second half of the period. Durations of the (a) sensor failures and (b) braking exceedances are noted in parentheses. the period than during the first half, thus revealing a strong hysteresis of reliance on ACC. For all non-failure periods, automation reliance did not significantly differ between display types (rain: $M=.68, \mathrm{SE}=0.07$; traffic: $M=.67$, $\mathrm{SE}=0.06$ ).

Analysis of the percent of time the ACC was engaged during failures showed that with and without use of the EID display, drivers relied on the ACC less as the magnitude of the failures increased. However, drivers also relied on the ACC more during the first half of the rain/ traffic periods than during the second half. This nonlinear reliance across time shows that reliance does not recover as quickly as reliability, a finding consistent with that of other researchers (Lee and Moray, 1994; Lewandowsky et al., 2000; Lee and See, 2004). Reliance results for failure and non-failure periods together provide a high-level representation of how often drivers use the ACC and in what operating condition: normal or failure. The next section presents a more detailed analysis that considers how quickly drivers intervene and assume control.

\subsection{Driver intervention}

Analysis of driver response to ACC failure, with measures of brake RT and THW at brake response, shows how effectively drivers intervened in switching from automatic to manual control. In rain drives, no statistically significant effects were observed for display type. There were also no significant differences between manual and ACC control for measures of mean brake RT to sensor failures and THW at those brake responses. Because drivers tended not to rely on the ACC during the rain, they initiated a total of only 46 brake responses with a nonsignificant difference in distribution of responses between display conditions, $\chi^{2}(1)=0.78, p=.376$.

Fig. 4 shows the effects of failure level on brake response for the traffic drives in which braking algorithm limits were exceeded. The magnitude of the exceedance influenced how quickly drivers responded with a brake press, $F(3,45)=$ $6.95, p<.001$. Drivers in the no-display condition took longer to respond as the failure level increased, whereas drivers in the EID-display condition reacted with a consistent RT across failure levels that was faster than in the no-display condition, $F(3,45)=3.75, p=.017$. Posthoc one-way ANOVAs showed that for the EID-display condition, the effect of the failure magnitude did not reach statistical significance, $F(3,28)=2.64, p=.07$, but for the no-display condition it did, $F(3,17)=3.57, p=.036$. These brake RT effects (Fig. 4a) were reflected in a shorter THW (Fig. 4b). Drivers in the no-display condition had reduced THWs compared to those with the EID display, $F(1,45)=16.4, p<.001$, and this difference increased across failure levels, $F(3,45)=3.37, p=.027)$. Note that the large variance in THW at brake response for failure level 1 in the no-display condition results from two divergent responses, which are indicated as x's in Fig. 4b. There was less variance in brake RT with the EID display 

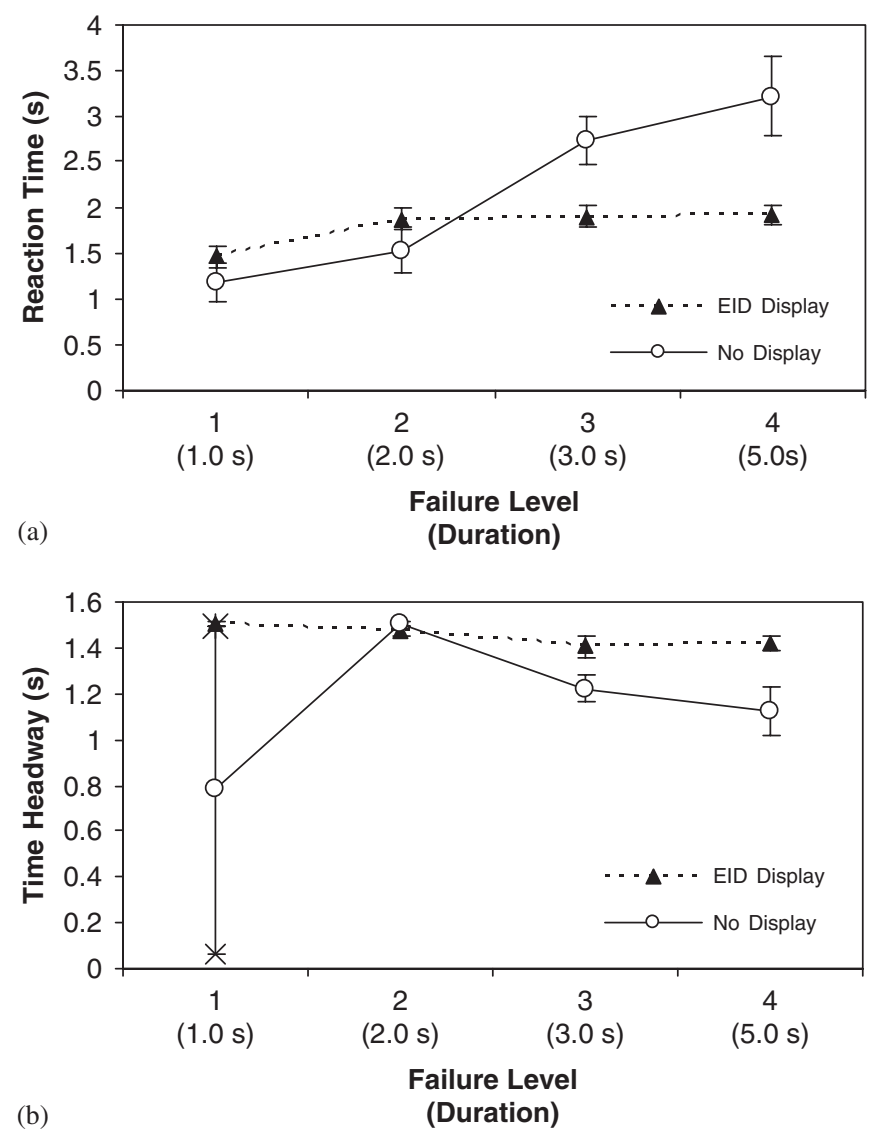

Fig. 4. (a) Mean brake RT for traffic periods by display type and failure level and (b) mean THW at brake response for traffic periods by display type and failure level. Durations of the braking exceedances are noted in parentheses.

than without the display (difference in variance: $F(9,10)=5.26, p<.01)$ and consequently less variance in the THW at brake response with the EID display than without, $F(9,10)=8.75, p<.01$. The longer THW at brake response for the EID-display condition led to fewer collisions compared to the no-display condition; there were no collisions for drivers with the EID display and five collisions for those with no display, $\chi^{2}(1)=5.0, p=.025$.

The RTs from Fig. 4a and the corresponding THWs at brake response from Fig. $4 \mathrm{~b}$ at the lower failure levels represent relatively few responses and are thus not representative of the entire subject population, representing instead the "conservative" drivers. Fig. 5 shows the total number of brake responses for each failure level, and indicates that the frequency of brake responses increased with increasing failure levels. Importantly, Fig. 5 shows the safety benefit of the EID display in prompting more brake responses to the braking algorithm exceedances than the no-display condition at each failure level, $\chi^{2}(1)=31.06$, $p<.0001$.

Analyses of the brake response shows that interventions with the EID display are more successful than without this display. For braking algorithm exceedances in which drivers initiated a brake response, the EID display enabled

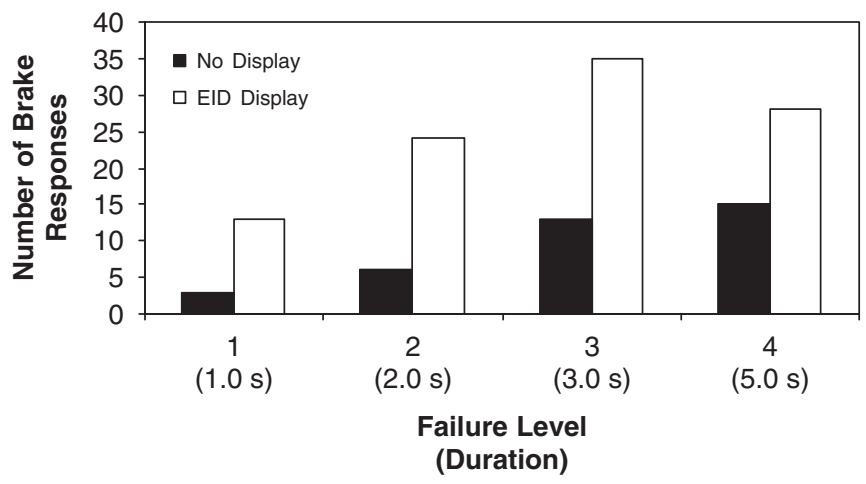

Fig. 5. Number of brake responses by display type and failure level. Durations of the braking exceedances are noted in parentheses.

more timely and frequent responses and increased response safety in terms of THW. The EID display prompted drivers to respond faster to failure events at a consistent THW regardless of failure level, whereas in the no-display condition, drivers were slower to respond to failures. The consistent brake response at a uniform $1.5 \mathrm{~s}$ THW indicates that the EID display helped drivers to detect deviations in THW resulting from braking algorithm exceedances at all failure levels. Drivers were able to determine the dynamic state of the ACC and thus rely more appropriately. This conclusion is consistent with the finding that when operators more accurately understand automation behavior they adopt a more effective attention allocation strategy (Wickens et al., 2000). Similarly, when informed of the capabilities of the automation operators rely more appropriately (Bisantz and Seong, 2001; Dzindolet et al., 2003).

For the sensor failures, low reliance on ACC during the rain periods led drivers to experience few failures - the ACC needed to be engaged for a sensor failure to occurand thus to a reduced number of brake responses to failures. It is likely that this low number of failures contributed to the lack of statistically significant benefit of the EID display to sensor failure brake response.

\subsubsection{Cluster analysis of intervention strategies}

Previous analyses describe driver brake response to automation failure; this section presents a cluster analysis of all driver interventions in which drivers transitioned from ACC to manual control with either a brake or a press of the "Off" button. This analysis clusters 163 interventions into eight sets according to six dependent variables (TTC, THW, driver velocity, LV velocity, brake pedal depression, and acceleration). Table 1 shows the state variables at the time of ACC disengagement (TTC, THW, driver velocity and LV velocity) and the response variables that qualify this disengagement (brake pedal depression and acceleration) for each of the eight clusters. Each of the 163 driver interventions was assigned to one of the eight clusters using the SAS $k$-means cluster analysis.

An examination of the state variables suggests possible motivators behind driver disengagement of the 
Table 1

Clusters and their characteristics that describe driver intervention strategies and distribution of cluster membership according to display and failure type (i.e. Rain condition; Traffic condition)

\begin{tabular}{|c|c|c|c|c|c|c|c|c|}
\hline & \multicolumn{4}{|c|}{ Safe intervention clusters } & \multicolumn{4}{|c|}{ Unsafe intervention clusters } \\
\hline & $\begin{array}{l}\text { 1: Rain- } \\
\text { proactive and } \\
\text { discomfort }\end{array}$ & $\begin{array}{l}\text { 2: Rain- } \\
\text { cautious and } \\
\text { early }\end{array}$ & $\begin{array}{l}\text { 3: Traffic- } \\
\text { cautious and } \\
\text { uncertain of } \\
\text { ACC } \\
\text { operation }\end{array}$ & $\begin{array}{l}\text { 4: Traffic- } \\
\text { proactive }\end{array}$ & $\begin{array}{l}\text { 5: Rain- } \\
\text { maximum } \\
\text { failures }\end{array}$ & $\begin{array}{l}\text { 6: Traffic- } \\
\text { maximum } \\
\text { failures }\end{array}$ & $\begin{array}{l}\text { 7: Rain- } \\
\text { hazards }\end{array}$ & $\begin{array}{l}\text { 8: Traffic- } \\
\text { hazards }\end{array}$ \\
\hline TTC (s) & 20.00 & 19.83 & 19.80 & 9.42 & 5.37 & 4.59 & 1.52 & 1.36 \\
\hline THW (s) & 1.90 & 1.94 & 1.39 & 1.33 & 0.79 & 1.14 & 1.14 & 0.79 \\
\hline Velocity (m/s) & 17.76 & 20.40 & 17.21 & 18.34 & 23.21 & 17.84 & 14.08 & 16.52 \\
\hline LV velocity $(\mathrm{m} / \mathrm{s})$ & 20.60 & 20.14 & 17.06 & 15.70 & 19.56 & 13.46 & 3.43 & 6.33 \\
\hline Brake pedal depression & 0.01 & 0.01 & 0.17 & 0.24 & 0.17 & 0.34 & 0.35 & 0.36 \\
\hline Acceleration (g) & 0.02 & -0.02 & -0.05 & -0.11 & -0.21 & -0.09 & -0.25 & -0.10 \\
\hline Total interventions & 10 & 33 & 11 & 11 & 9 & 31 & 23 & 35 \\
\hline Brake pedal depression & 0 & 0 & 5 & 7 & 4 & 30 & 22 & 35 \\
\hline No display & 0 & 0 & 4 & 2 & 2 & 14 & 13 & 17 \\
\hline EID display & 0 & 0 & 1 & 5 & 2 & 16 & 9 & 18 \\
\hline Button press & 10 & 33 & 6 & 4 & 5 & 1 & 1 & 0 \\
\hline No display & 5 & 16 & 0 & 0 & 3 & 0 & 0 & 0 \\
\hline EID display & 5 & 17 & 6 & 4 & 2 & 1 & 1 & 0 \\
\hline Rain condition & 8 & 29 & 3 & 1 & 9 & 0 & 22 & 2 \\
\hline No display & 5 & 13 & 0 & 0 & 5 & 0 & 13 & 0 \\
\hline EID display & 3 & 16 & 3 & 1 & 4 & 0 & 9 & 2 \\
\hline Traffic condition & 2 & 4 & 8 & 10 & 0 & 31 & 1 & 33 \\
\hline No display & 0 & 3 & 4 & 2 & 0 & 14 & 0 & 17 \\
\hline EID display & 2 & 1 & 4 & 8 & 0 & 17 & 1 & 16 \\
\hline
\end{tabular}

ACC - these define the labels of each of the eight clusters in Table 1. In Cluster 1, drivers disengaged the ACC when there was a large TTC (20.00 s) and THW (1.90 s); eight out of ten of these interventions occurred during rain conditions with a mean rain level of .707 , where 1.0 is the maximum intensity of rain and 0.0 is no rain. Thus, heavy rain motivated a proactive strategy of disengagement. Drivers disengaged before the maximum failure level, likely because they were uncomfortable with the lack of visibility caused by the heavy rain. The positive acceleration value reflects that in some of the interventions, drivers had their foot resting on the accelerator pedal. In Cluster 2, drivers again disengaged ACC when there was a large TTC $(19.83 \mathrm{~s})$ and THW (1.94s). However, with this group, a mean rain level of only .267 for 29 out of the 33 interventions during rain conditions may reflect a more cautious strategy of early disengagement. In Clusters 3 and 4 , drivers responding to $\mathrm{LV}$ decelerations intervened with a TTC greater than $9.0 \mathrm{~s}$ and a THW greater than $1.30 \mathrm{~s}$. Eight out of 11, and ten out of 11 interventions occurred during traffic conditions for Clusters 3 and 4, respectively. The relatively large TTC of Cluster 3 represents a cautious strategy of disengagement. Drivers also demonstrated a proactive strategy in Cluster 4 as they disengaged before the maximum LV deceleration events (or failure levels). In contrast to Clusters 1-4, drivers in Clusters 5-8 intervened from necessity. Drivers disengaged the ACC in Clusters 5 and 6 during the high-level rain sensor failures and traffic braking algorithm exceedances, respectively. Intense LV deceleration events prompted driver interventions in Clusters 7 and $8 ; 22$ out of 23 interventions occurred during rain conditions in Cluster 7 , and 33 out of 35 occurred during traffic conditions in Cluster 8. Interestingly, interventions were clustered such that sensor failures and braking algorithm exceedances were placed in nearly homogeneous groups.

The clusters in Table 1 are categorized into safe and unsafe interventions based on the combination of a TTC of less than 20 and a THW of less than 10. TTC and THW indicate driving safety in following a LV (Vogel, 2003), and in part, the amount of degradation in ACC performance drivers allowed before intervening (a fully functional ACC system, in this experiment, maintained an approximate $1.5 \mathrm{~s}$ THW with a large TTC). Brake pedal depression and acceleration characterize the severity of the brake response at disengagement. Not surprisingly, the mean brake pedal depression was lower for the safe-intervention Clusters 1-4 (0.11) compared to the unsafe-intervention Clusters 5-8 $(0.31)$.

The cluster membership reveals the effect of display type and failure type on driver intervention strategies. As shown in the cluster descriptions and in Table 1, failure type significantly influenced driver intervention strategy, $\chi^{2}(7)=39.42, p<.0001$. Note that Cluster 5 consists 
entirely of driver interventions in rain conditions and Cluster 6 entirely of driver interventions in traffic conditions. Interestingly, within the safe-intervention clusters, drivers in the rain clusters (Clusters 1 and 2) intervened with a larger THW using the "Off" button than in the traffic clusters (Clusters 3 and 4), where drivers used the brake pedal, $\chi^{2}(1)=25.26, p<.0001$. This difference in intervention type was partly due to the way in which the LV braking and failure events were operationalized in the rain and traffic conditions. The more gradual LV deceleration pattern in the rain condition allowed for more time to observe and respond to the $\mathrm{LV}$, as is evident in the larger THWs. However, this difference in intervention type also reflects drivers' understanding of the ACC. Whereas a button press signals an intended shift in reliance, a brake press can indicate either an intended or forced disengagement. For the high traffic safe-intervention clusters, display type differentiates intervention; drivers disengaged ACC more readily with a button press than with a brake press in the EID-display condition, and intervened more often with brake presses than button presses in the no-display condition, $\chi^{2}(1)=7.80, p<.01$ (Cluster 3$), \chi^{2}(1)=5.29$, $p=.022$ (Cluster 4).

Display type did not have a statistically significant effect overall on driver intervention strategy, $\chi^{2}(7)=5.41$, $p=.610$, or on the proportion of safe and unsafe interventions, $\chi^{2}(1)=1.86, p=.172$. However, the proactive driver intervention strategy in traffic conditions (Cluster 4) depended on display type, $\chi^{2}(1)=4.46$, $p=.035$, revealing a potential safety benefit of the EID display.

The cluster analysis for traffic conditions showed that the EID led drivers to adopt a more proactive disengagement strategy compared to the no-display condition. Drivers with the EID display intervened more frequently before the maximum LV deceleration events or failure levels were reached. A proactive disengagement strategy indicates that drivers relied more appropriately on the ACC. Intervention type (i.e. button press or brake press) also showed that EID promotes a proactive disengagement strategy. Drivers without the EID display were more likely to disengage ACC with a brake press, that is, they were forced to intervene due to the criticality of the situation.

\subsection{Benefits of EID in manual control}

Previous analyses described driver response to ACC failures due to increased rain and traffic. This section focuses on the benefit of the EID display in manual control in response to LV braking. Drivers manually controlled the vehicle during manual drives and during the periods of the ACC drives when the system was not engaged.

The mean brake RTs for the rain periods are shown in Fig. 6a, which presents manual and ACC-not-engaged conditions by display type; the long reaction times are reflective of the gradual LV deceleration pattern. Across these manual control conditions, display type showed a

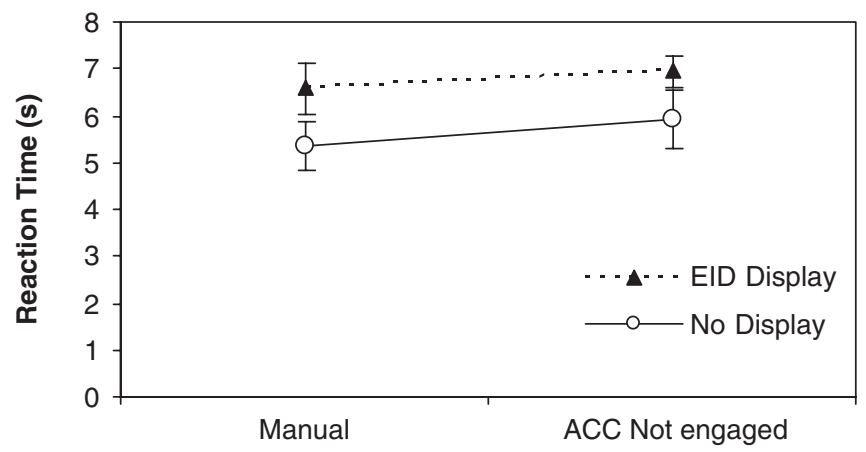

(a) Automation Level

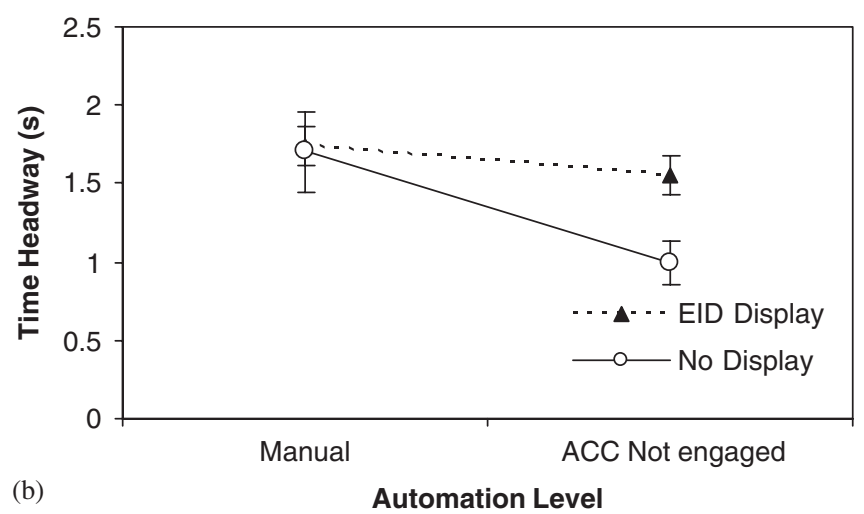

Fig. 6. (a) Mean brake RT for rain periods by display type and automation level and (b) mean THW at brake response for rain periods by display type and automation level.

marginally significant effect for brake RT, $F(1,22)=4.21$, $p=.052$, with drivers with the EID delaying brake response to LV velocity changes compared to those with no display. This longer time to initiate a brake response with the EID display did not result in a significant reduction in the THW, $F(1,22)=1.1, p=.305$ (see Fig. 6b). Drivers allowed a shorter THW when ACC was available but not engaged in the no-display condition compared to the EID display condition, $F(1,12)=4.62$, $p=.053$, a marginally significant interaction. A more consistent THW is apparent in manual control for the EID display compared to the no-display condition, as is evidenced by smaller variance in THW at brake response for the EID display condition, $F(11,11)=3.77, p=.019$.

There were no statistically significant differences between display types or between manual control situations and situations in which ACC was not engaged, as measured by brake RT and THW at brake response in traffic periods. It is important to note, however, that the EID display reduced the variance in THW at brake response in manual control compared to the no-display condition, $F(11,11)=3.15, p=.035$.

The EID display promoted more consistent car-following performance. Though brake RT to LV decelerations did not depend on the EID display, drivers with the EID display initiated their brake responses at THWs that allowed them to maintain the instructed $1.5 \mathrm{~s}$ THW. This 
benefit in manual control suggests that the continuous display supports drivers beyond merely indicating failures from the emergent triangle shape; drivers were more aware of the roadway, and thus better able to monitor headway. They were able to associate the meaning of the EID display with the LV behavior and with the position of the shape within the display to maintain a consistent THW. This benefit to car-following behavior may translate to an improved response to hazardous situations in manual control and merits further investigation. If such an effect was found, the EID approach to informing drivers might be an important alternative to the more prevalent approach of warning drivers (Parasuraman et al., 1997; Lee et al., 2004).

\subsection{Secondary task performance}

The secondary task provides an indirect measure of the demands of the driving task. Some of the responses to questions were significantly delayed; these outliers, responses larger than three standard deviations from the mean, were removed (such responses constituted less than $2 \%$ of the data).

During the rain periods, drivers with the EID display $(M=2.86, \quad \mathrm{SE}=0.17)$ reacted more quickly to the questions compared to those with no display $(M=4.06$, $\mathrm{SE}=0.30), F(1,22)=5.14, p=.033$. During the traffic periods, drivers showed a similar trend (EID display: $M=3.22, \mathrm{SE}=0.24$; no display: $M=4.54, \mathrm{SE}=0.35$ ), $F(1,22)=3.94, \quad p=.059$. Differences in RT between manual and ACC control did not reach statistical significance, nor was there an interaction with display type for either failure type. The correlation of brake RT to LV decelerations and question RT in rain periods both with and without use of the EID display were non-significant, $r(23)=-.04, p=.86, r(26)=-.09, p=.63$, respectively. The same was true of the traffic periods both with and without use of the EID display, $r(18)=.29, p=.21$, $r(19)=-.19, p=.41$, respectively. The faster RT to questions in the EID display condition compared to those in the no-display condition of both the rain and traffic periods did not result in a tradeoff of accuracy as there were no statistically significant effects for accuracy (rain: $M=$ $71.24 \%, \mathrm{SE}=2.17 \%$; traffic: $M=71.45 \%, \mathrm{SE}=2.51 \%$ ).

The lack of correlation between brake RT to LV decelerations and question RT shows there was no tradeoff in task allocation between driving and the secondary tasks. Drivers had greater capacity to perform the secondary task with the EID display than with no display, which was reflected in the faster RT without decrement to the brake reaction or to the accuracy of question responses. These results suggest that providing continuous information regarding the state of the automation does not necessarily overburden the operator. This finding contrasts with the display-by-exception approach and focus on imminent crash warnings, which assume that continuous information regarding reliable automation will undermine operators' ability to attend to other tasks (Palmer and Abbott, 1994; Kiefer et al., 2005).

\subsection{Limitations}

Because this study was conducted in a driving simulator, the benefits of the EID display might not translate to benefits in actual driving situations. In particular, while it is expected that the EID display will help in actual driving situations, in which drivers encounter great variability, by informing drivers of the behavior of the ACC in each situation, this study included only a small subset of the possible situations, defined by the braking profiles and sensor failures. However, care was taken to ensure that both the use and operation of the ACC mimicked the real world. Specifically, drivers were able to engage and disengage the ACC in accord with their assessment of the appropriateness of using the system. Further, the conditions of heavy rain and traffic, which induced the differential failures of the ACC, were based on current descriptions of ACC.

Drivers' ability to engage or disengage the automation led to an unequal number of failures per participant, as failures were only experienced when the ACC was engaged. However, it was necessary to sacrifice control in this way in order to observe driver intervention strategies and to measure the percent reliance based on interaction of the environment with ACC function.

Another limitation of this study concerns the particular instruction given to participants to maintain a $1.5 \mathrm{~s}$ THW. Although they were not specifically told that the ACC was imperfect, this instruction may have biased participants to focus on the ACC's maintenance of the $1.5 \mathrm{~s}$ THW where the failure to maintain this THW was an indicator of failure both in the rain and traffic conditions. Drivers may therefore have detected more failures more quickly than they would have with an instruction to maintain a 'comfortable' following distance or to avoid cut-ins. However, it is unlikely that this emphasis biased the results toward the EID display because drivers in both display conditions were provided with the same instructions.

Finally, the number of failures in each drive, 44, may have increased drivers' sensitivity to failure events. The high failure rate in this experiment represents a difficult tradeoff between collecting data regarding drivers' response to several failures in a cost effective manner and the need to include a realistic failure rate. Although the number and pattern of failures were designed to model real-world degradation of sensors and the limits of the ACC braking authority associated with rain and traffic, respectively, it is unlikely that such a large number of failures would actually occur in any single 20 -min period. Compared to actual driving conditions, drivers in this experiment received relatively little exposure to 'normal' driving conditions in which ACC would operate consistently. Longer drives with infrequent ACC failures may 
lead drivers to rely on the ACC more heavily. In other domains, automation that fails after long periods of consistent performance leads operators to neglect automation failures (Parasuraman et al., 1993; Singh et al., 1997). As a consequence, this experiment may underestimate the benefits of EID in actual driving situations where ACC failures are rare.

\section{Conclusion}

The EID display enhanced driver response in both manual control of car following and in responding to ACC failures. EID provided a continuous indication of automation and roadway state that helped to make the subtle cues associated with braking behavior of the LV more salient, prompting appropriate reliance in terms of the detection and response to automation failure when braking limits were exceeded. The EID display did not provide a benefit in rain situations, however, in part because the failures were unrelated to the LV's behavior. The benefit in manual control and superior performance with the secondary task demonstrates that the EID diminished the demands of monitoring the LV. These results show that even in a domain that makes heavy demands on visual attention, continuous information can enhance performance, even when presented in a somewhat complex graphical display. The EID display reduced the demands of the driving task, freed capacity to complete other tasks, and enhanced automation management. EID provides a useful alternative to the display-by-exception approach that is often advocated in supporting automation management (Palmer and Abbott, 1994).

We should stress that not just any complex representation of continuous information will enhance driving performance. The triangular image created from the THW, TTC $^{-1}$, and range rate provided a salient indicator of hazardous braking events, evident from the faster, consistent, and more frequent brake responses. This emergent feature made a complex combination of variables accessible to drivers (Vicente, 1999; Hajdukiewicz and Vicente, 2002). However, for sensor degradation, as experienced by the drivers in the rain condition, color dilution was not an effective cue; this cue masked the indication of sensor failure - a faltering update rate. Feature degradation, a proposed graphical means to display uncertainty of information in which pixel resolution is reduced, might provide a more effective cue of the reliability of the displayed information (Finger and Bisantz, 2002), and might enhance the utility of the EID in the rain conditions.

The differential benefit of the EID display between the two failure types mirrors the conflict in the literature regarding the benefits (Rakha et al., 2001; Ioannou and Stefanovic, 2005; Lee et al., in press) and disbenefits of ACC (Stanton et al., 1997; Stanton and Young, 1998; Hoedemaeker, 2000; Marsden et al., 2001; Rudin-Brown and Parker, 2004; Young and Stanton, 2004). This study suggests such conflicts may result both from differing experimental paradigms for comparing manual and ACC control (Young and Stanton, 2004), and from exposing drivers to failures without regard to the environmental context in which those failures would occur (Bagheri and Jamieson, 2004).

\section{Acknowledgments}

This research was supported by Nissan Motor Co., Ltd., with special thanks to Erwin Boer, project coordinator, and by a fellowship from the US Department of Transportation Federal Highway Administration/Universities and Grants Program. Teresa Lopes of the Public Policy Center at the University of Iowa provided extensive help with technical editing and document preparation.

\section{References}

Bagheri, N., Jamieson, G.A., 2004. The impact of context-related reliability on automation failure detection and scanning behavior. In: IEEE International Conference on Systems, Man, and Cybernetics, pp. 212-217.

Bisantz, A.M., Seong, Y., 2001. Assessment of operator trust in and utilization of automated decision-aids under different framing conditions. International Journal of Industrial Ergonomics 28 (2), 85-97.

Davis, L.C., 2004. Effect of adaptive cruise control on traffic flow. Physical Review E 69 (6), 1-8.

Dzindolet, M.T., Peterson, S.A., Pomranky, R.A., Pierce, L.G., Beck, H.P., 2003. The role of trust in automation reliance. International Journal of Human-Computer Studies 58 (6), 697-718.

Fancher, P., Bareket, Z., Ervin, R., 2001. Human-centered design of an Acc-with-braking and forward-crash-warning system. Vehicle System Dynamics 36 (2-3), 203-223.

Finger, R., Bisantz, A.M., 2002. Utilizing graphical formats to convey uncertainty in a decision-making task. Theoretical Issues in Ergonomics Science 3 (1), 1-25.

Goodrich, M.A., Boer, E.R., 2003. Model-based human-centered task automation: a case study in ACC system design. IEEE Transactions on Systems, Man, and Cybernetics Part A-Systems and Humans 33 (3), 325-336.

Hajdukiewicz, J.R., Vicente, K.J., 2002. Designing for adaptation to novelty and change: functional information, emergent feature graphics, and higher-level control. Human Factors 44 (4), 592-610.

Hills, B.L., 1980. Vision, visibility, and perception in driving. Perception 9 (2), 183-216.

Hoedemaeker, M., 2000. Driving behaviour with ACC and the acceptance by individual drivers. In: IEEE Intelligent Transportation Systems Proceedings, 2000 IEEE, pp. 506-509.

Hoffmann, E.R., Mortimer, R.G., 1994. Drivers estimates of time to collision. Accident Analysis and Prevention 26 (4), 511-520.

Hoffmann, E.R., Mortimer, R.G., 1996. Scaling of relative velocity between vehicles. Accident Analysis and Prevention 28 (4), 415-421.

Ioannou, P.A., Stefanovic, M., 2005. Evaluation of ACC vehicles in mixed traffic: Lane change effects and sensitivity analysis. IEEE Transactions on Intelligent Transportation Systems 6 (1), 79-89.

Kiefer, R.J., LeBlanc, D.J., Flannagan, C.A., 2005. Developing an inverse time-to-collision crash alert timing approach based on drivers' lastsecond braking and steering judgments. Accident Analysis and Prevention 37 (2), 295-303.

Lee, J.D., Moray, N., 1994. Trust, self-confidence, and operators' adaptation to automation. International Journal of Human-Computer Studies 40, 153-184. 
Lee, J.D., See, K.A., 2004. Trust in technology: designing for appropriate reliance. Human Factors 46 (1), 50-80.

Lee, J.D., Hoffman, J.D., Hayes, E., 2004. Collision warning design to mitigate driver distraction. In: Proceedings of CHI. ACM, New York, pp. 65-72.

Lee, J.D., McGehee, D.V., Brown, T.L., Marshall, D.C. Effects of adaptive cruise control and alert modality on driver performance. Transportation Research Record, in press.

Lewandowsky, S., Mundy, M., Tan, G., 2000. The dynamics of trust: comparing humans to automation. Journal of Experimental Psychology-Applied 6 (2), 104-123.

Marsden, G., McDonald, M., Brackstone, M., 2001. Towards an understanding of adaptive cruise control. Transportation Research Part C-Emerging Technologies 9 (1), 33-51.

Nilsson, L., 1995. Safety effects of adaptive cruise control in critical traffic situations. In: Proceedings of the Second World Congress on Intelligent Transport Systems: 'Steps Forward', III (Yokohama: VERTIS), pp. 1254-1259.

Palmer, M.T., Abbott, K.H., 1994. Effects of expected-value information and display format on recognition of aircraft subsystem abnormalities: NASA Technical Paper 3395.

Parasuraman, R., Riley, V., 1997. Humans and automation: use, misuse, disuse, abuse. Human Factors 39 (2), 230-253.

Parasuraman, R., Molloy, R., Singh, I., 1993. Performance consequences of automation-induced "complacency". International Journal of Aviation Psychology 3 (1), 1-23.

Parasuraman, R., Hancock, P.A., Olofinboba, O., 1997. Alarm effectiveness in driver-centred collision-warning systems. Ergonomics 40 (3), 390-399.

Rakha, H., Hankey, J., Patterson, A., Van Aerde, M., 2001. Field evaluation of safety impacts of adaptive cruise control. Its Journal 6 (3), 225-259.

Reyes, M.L., Lee, J.D., 2004. The influence of IVIS distractions on tactical and control levels of driving performance. In: Proceedings of the 48th Annual Meeting of the Human Factors and Ergonomics Society, vol. 2. Human Factors and Ergonomics Society, Santa Monica, CA, pp. 2369-2373.

Riley, V., 1994. Human use of automation. Unpublished doctoral dissertation. University of Minnesota, Minneapolis.

Rudin-Brown, C.M., Parker, H.A., 2004. Behavioural adaptation to adaptive cruise control (ACC): implications for preventive strategies. Transportation Research Part F-Traffic Psychology and Behaviour 7 (2), 59-76.
Seppelt, B.D., Lees, M.N., Lee, J.D., 2005a. Driver distraction and reliance: Adaptive cruise control in the context of sensor reliability and algorithm limits. In: Proceedings of the Third International Driving Symposium on Human Factors in Driver Assessment, Training and Vehicle Design, 2005 Driving Assessment, pp. 255-261.

Seppelt, B.D., Lees, M.N., Lee, J.D., Stoner, H.A., Brown, M., Hoffman, J.D., 2005b. Integrating ecological interface design into the engineering design process for vehicles. Nissan Final Report Year 3, University of Iowa, Iowa City.

Singh, I.L., Molloy, R., Parasuraman, R., 1997. Automation-induced monitoring inefficiency: role of display location. International Journal of Human-Computer Studies 46 (1), 17-30.

Stanton, N.A., Marsden, P., 1996. From fly-by-wire to drive-by-wire: safety implications of automation in vehicles. Safety Science 24 (1), 35-49.

Stanton, N.A., Young, M.S., 1998. Vehicle automation and driving performance. Ergonomics 41 (7), 1014-1028.

Stanton, N.A., Young, M., McCaulder, B., 1997. Drive-by-wire: the case of driver workload and reclaiming control with adaptive cruise control. Safety Science 27 (2), 149-159.

Stoner, H.A., Wiese, E.E., Lee, J.D., 2003. Applying ecological interface design to the driving domain: the results of an abstraction hierarchy analysis. In: Human Factors and Ergonomics Society 47th Annual Meeting.

Vicente, K.J., 1999. Cognitive Work Analysis: Towards Safe, Productive, and Healthy Computer-based Work. Lawrence Erlbaum Associates, Mahwah, NJ.

Vicente, K.J., 2002. Ecological interface design: progress and challenges. Human Factors 44 (1), 62-78.

Vicente, K.J., Rasmussen, J., 1992. Ecological interface design: theoretical foundations. IEEE Transactions on Systems, Man, and Cybernetics SCM-22 (4), 589-606.

Vogel, K., 2003. A comparison of headway and time to collision as safety indicators. Accident Analysis \& Prevention 35, 427-433.

Wickens, C.D., 1992. Engineering Psychology and Human Performance. Harper Collins, New York, NY.

Wickens, C.D., Gempler, K., Morphew, M.E., 2000. Workload and reliability of predictor displays in aircraft traffic avoidance. Transportation Human Factors 2 (2), 99-126.

Young, M.S., Stanton, N.A., 2004. Taking the load off: investigations of how adaptive cruise control affects mental workload. Ergonomics 47 (9), 1014-1035. 